\title{
On the number of maximal independent sets in a graph
}

\author{
David R. Wood $\|^{\dagger}$ \\ Department of Mathematics and Statistics, The University of Melbourne, Melbourne, Australia \\ received $17^{\text {th }}$ August 2011, accepted $30^{\text {th }}$ August 2011.
}

Miller and Muller (1960) and independently Moon and Moser (1965) determined the maximum number of maximal independent sets in an $n$-vertex graph. We give a new and simple proof of this result.

MSC: 05C30 Enumeration in graph theory; 05C69 Dominating sets, independent sets, cliques

Keywords: graph, independent sets

Let $G$ be a (simple, undirected, finite) graph. A set $S \subseteq V(G)$ is independent if no edge of $G$ has both its endpoints in $S$. An independent set $S$ is maximal if no independent set of $G$ properly contains $S$. Let $\operatorname{MIS}(G)$ be the set of all maximal independent sets in $G$. Miller and Muller (1960) and Moon and Moser (1965) independently proved that the maximum, taken over all $n$-vertex graphs $G$, of $|\operatorname{MIS}(G)|$ equals

$$
g(n):=\left\{\begin{array}{lll}
3^{n / 3} & \text { if } n \equiv 0 & (\bmod 3) \\
4 \cdot 3^{(n-4) / 3} & \text { if } n \equiv 1 & (\bmod 3) \\
2 \cdot 3^{(n-2) / 3} & \text { if } n \equiv 2 & (\bmod 3)
\end{array}\right.
$$

This result is important for various reasons. For example, $g(n)$ bounds the time complexity of various algorithms that output all maximal independent sets (Bron and Kerbosch, 1973; Lawler et al., 1980, Tsukiyama et al., 1977, Tomita et al., 2006, Johnson et al., 1988, Eppstein. 2003, Eppstein et al., 2010). Here we give a new and simple proof of this upper bound on $|\mathrm{MIS}(G)|$.

Theorem 1 ((Miller and Muller, 1960; Moon and Moser, 1965)) For every n-vertex graph $G$,

$$
|\operatorname{MIS}(G)| \leq g(n) .
$$

Proof: We proceed by induction on $n$. The base case with $n \leq 2$ is easily verified. Now assume that $n \geq 3$. Let $G$ be a graph with $n$ vertices. Let $d$ be the minimum degree of $G$. Let $v$ be a vertex of degree

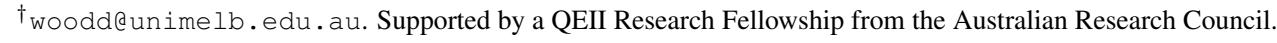

1365-8050 (C) 2011 Discrete Mathematics and Theoretical Computer Science (DMTCS), Nancy, France 
$d$ in $G$. Let $N[v]$ be the closed neighbourhood of $v$. If $I \in \operatorname{MIS}(G)$ then $I \cap N[v] \neq \emptyset$, otherwise $I \cup\{v\}$ would be an independent set. Moreover, if $w \in I \cap N[v]$ then $I \backslash\{w\} \in \operatorname{MIS}(G-N[w])$. Thus

$$
|\operatorname{MIS}(G)| \leq \sum_{w \in N_{G}[v]}\left|\operatorname{MIS}\left(G-N_{G}[w]\right)\right|
$$

Since $\operatorname{deg}(w) \geq d$ and $g$ is non-decreasing, by induction,

$$
|\operatorname{MIS}(G)| \leq(d+1) \cdot g(n-d-1) .
$$

Note that

$$
4 \cdot 3^{(n-4) / 3} \leq g(n) \leq 3^{n / 3}
$$

If $d \geq 3$ then

$$
|\operatorname{MIS}(G)| \leq(d+1) \cdot 3^{(n-d-1) / 3} \leq 4 \cdot 3^{(n-4) / 3} \leq g(n) .
$$

If $d=2$ then

$$
|\operatorname{MIS}(G)| \leq 3 \cdot g(n-3)=g(n) .
$$

If $d=1$ and $n \equiv 1(\bmod 3)$ then since $n-2 \equiv 2(\bmod 3)$,

$$
\operatorname{MIS}(G) \leq 2 \cdot g(n-2) \leq 2 \cdot 2 \cdot 3^{(n-2-2) / 3}=4 \cdot 3^{(n-4) / 3}=g(n) .
$$

If $d=1$ and $n \equiv 0(\bmod 3)$ then since $n-2 \equiv 1(\bmod 3)$,

$$
\operatorname{MIS}(G) \leq 2 \cdot g(n-2) \leq 2 \cdot 4 \cdot 3^{(n-2-4) / 3}<3^{n / 3}=g(n) .
$$

If $d=1$ and $n \equiv 2(\bmod 3)$ then since $n-2 \equiv 0(\bmod 3)$,

$$
\operatorname{MIS}(G) \leq 2 \cdot g(n-2) \leq 2 \cdot 3^{(n-2) / 3}=g(n) .
$$

This proves that $|\operatorname{MIS}(G)| \leq g(n)$, as desired.

For completeness we describe the example by Miller and Muller (1960) and Moon and Moser (1965) that proves that Theorem 1 is best possible. If $n \equiv 0(\bmod 3)$ then let $M_{n}$ be the disjoint union of $\frac{n}{3}$ copies of $K_{3}$. If $n \equiv 1(\bmod 3)$ then let $M_{n}$ be the disjoint union of $K_{4}$ and $\frac{n-4}{3}$ copies of $K_{3}$. If $n \equiv 2$ $(\bmod 3)$ then let $M_{n}$ be the disjoint union of $K_{2}$ and $\frac{n-2}{3}$ copies of $K_{3}$. Observe that $\left|\operatorname{MIS}\left(M_{n}\right)\right|=g(n)$.

Note that Vatter (2011) gave another proof of Theorem 1, and also described a connection between this result and the question, "What is the largest integer that is the product of positive integers with sum $n$ ?" Also note that Dieter Kratsch proved that $|\operatorname{MIS}(G)| \leq 3^{n / 3}$ using a similar proof to that presented here; see Gaspers (2010, page 177). Thanks to the authors of (Vatter, 2011, Gaspers, 2010) for pointing out these references. 
On the number of maximal independent sets in a graph

\section{References}

C. Bron and J. Kerbosch. Finding all cliques of an undirected graph. Comm. ACM, 16(9):575-577, 1973. doi:10.1145/362342.362367.

D. Eppstein. Small maximal independent sets and faster exact graph coloring. J. Graph Algorithms Appl., 7(2):131-140, 2003. http://jgaa.info/accepted/2003/Eppstein2003.7.2.pdf.

D. Eppstein, M. Löffler, and D. Strash. Listing all maximal cliques in sparse graphs in near-optimal time. In O. Cheong, K.-Y. Chwa, and K. Park, editors, Proc. of 21st International Symposium on Algorithms and Computation (ISAAC 2010), Lecture Notes in Computer Science 6056:403-414, 2010. doi:10.1007/978-3-642-17517-6_36.

S. Gaspers. Exponential Time Algorithms: Structures, Measures, and Bounds. VDM Verlag Dr. Müller, 2010. http://www.kr.tuwien.ac.at/drm/gaspers/SergeBookETA2010_screen. $\mathrm{pdf}$

D. S. Johnson, M. Yannakakis, and C. H. Papadimitriou. On generating all maximal independent sets. Inform. Process. Lett., 27(3):119-123, 1988. doi:10.1016/0020-0190 (88) 90065-8.

E. L. Lawler, J. K. Lenstra, and A. H. G. R. Kan. Generating all maximal independent sets: NP-hardness and polynomial-time algorithms. SIAM J. Comput., 9(3):558-565, 1980. doi:10.1137/0209042.

R. E. Miller and D. E. Muller. A problem of maximum consistent subsets. IBM Research Report RC-240, Thomas J. Watson Research Center, New York, USA, 1960.

J. W. Moon and L. Moser. On cliques in graphs. Israel J. Math., 3:23-28, 1965. doi:10.1007/BF02760024

E. Tomita, A. Tanaka, and H. Takahashi. The worst-case time complexity for generating all maximal cliques and computational experiments. Theoret. Comput. Sci., 363(1):28-42, 2006. doi:10.1016/j.tcs.2006.06.015.

S. Tsukiyama, M. Ide, H. Ariyoshi, and I. Shirakawa. A new algorithm for generating all the maximal independent sets. SIAM J. Comput., 6(3):505-517, 1977. doi:10.1137/0206036.

V. Vatter. Maximal independent sets and separating covers. American Mathematical Monthly, 118:418423, 2011. http://www.math.ufl.edu/ vatter/publications/sss-mis/ 
